\title{
Degree of hippocampal atrophy is not related to a history of febrile seizures in patients with proved hippocampal sclerosis
}

\author{
Simon P C Bower, Christine J Kilpatrick, Simon J Vogrin, Kevin Morris, Mark J Cook
}

\begin{abstract}
Objectives-To examine the degree of hippocampal atrophy in patients with temporal lobe epilepsy and proved hippocampal sclerosis to determine whether or not patients with febrile seizures have more severe hippocampal atrophy. To determine whether or not there is a relation between age of seizure onset, duration of temporal lobe epilepsy, or seizure frequency, and severity of hippocampal atrophy.

Methods-Hippocampal volumes were measured from volumetrically acquired MR images in 77 consecutive surgical patients with temporal lobe epilepsy (37 febrile seizures (FS),+ 40 FS-) with proved hippocampal sclerosis, and compared with 98 controls.

Results-Ipsilateral and contralateral hippocampal volumes were not significantly different between the FS+ and FSgroups. There was no difference in the age of onset of habitual seizures, duration of epilepsy, or age at the time of surgery, between these groups. No clinically significant correlations were found between hippocampal volumes and age of onset of first non-febrile seizure, duration of temporal lobe epilepsy, or complex partial and secondarily generalised seizure frequency, in patients with and without febrile seizures.

Conclusions-Although febrile seizures was associated with hippocampal sclerosis in $48 \%$ of patients in this surgical series, the degree of MRI determined hippocampal atrophy was not related to a history of such seizures. The results do not support the view that febrile seizures cause more severe hippocampal sclerosis and are consistent with the hypothesis that hippocampal sclerosis is a pre-existing abnormality. (F Neurol Neurosurg Psychiatry 2000;69:733-738)
\end{abstract}

Keywords: temporal lobe epilepsy; hippocampal sclerosis; febrile seizures; magnetic resonance imaging

Hippocampal sclerosis is the most common pathology in intractable temporal lobe epilepsy, occurring in $50 \%-70 \%$ of patients. ${ }^{1}$ The percentage of patients having febrile seizures in surgical series of hippocampal sclerosis varies between $20 \%$ and $67 \%$, and these are usually, but not always prolonged or complex febrile seizures. ${ }^{2-9}$ However prospect cohort studies confirm that intractable temporal lobe epilepsy is only a rare outcome in children with complex febrile seizures. ${ }^{10-12}$ Therefore the vast majority of cases of febrile seizures are not associated with hippocampal sclerosis, and on average half the patients with hippocampal sclerosis, at least in surgical series, have no history of these seizures.

Nevertheless, several hypotheses are proposed to explain the relation between febrile seizures, hippocampal sclerosis, and temporal lobe epilepsy. hippocampal sclerosis may be acquired before the seizures as a result of a prior insult, ${ }^{11}$ the seizures may produce injury that evolves into hippocampal sclerosis, ${ }^{13}{ }^{14}$ or a pre-existing developmental abnormality may predispose to febrile seizures, which then may cause additional hippocampal damage. ${ }^{15}$

Animal models suggest that the immature hippocampus is relatively resistant to hippocampal injury, ${ }^{16}$ whereas rats with experimentally induced dysgenesis are more susceptible to hyperthermia induced seizures and hippocampal injury. ${ }^{17} 18$

Brain MR studies suggesting that patients with temporal lobe epilepsy with a history of febrile seizures have more severe hippocampal atrophy ipsilateral to the seizure focus than those without seizures might be interpreted as supporting the hypothesis that febrile seizures produce injury that evolves into hippocampal sclerosis. ${ }^{219-23}$ Alternatively, if febrile seizures occur in the setting of a previously abnormal hippocampus, it might be expected that the degree of MR determined hippocampal atrophy would be similar in patients with and without febrile seizures. No previous studies have included only patients with pathologically established hippocampal sclerosis.

We therefore studied 77 patients with temporal lobe epilepsy and proved hippocampal sclerosis to determine whether or not patients with febrile seizures have more severe hippocampal atrophy. We also correlated hippocampal volumes with clinical variables to determine whether or not there is a relation between age of seizure onset, temporal lobe epilepsy duration, or seizure frequency, and severity of hippocampal atrophy.

\section{Methods}

PATIENTS

The study population comprised 77 patients with temporal lobe epilepsy selected for surgery on the basis of intractable, drug resistant complex partial seizures and confirmatory video-EEG seizure lateralisation. There were 46 female and 31 male patients, mean age 37.4 
(SD 9.0) (range 20-55) years. A left temporal lobectomy was performed in 44 patients (23 female, 21 male) and a right temporal lobectomy in 33 patients (23 female, 10 male). Pathology confirmed hippocampal sclerosis in all patients, as defined by $>50 \%$ neuronal loss in the CA1 subfield of the hippocampus.

Clinical features were recorded prospectively on admission for video-EEG monitoring and entered into our comprehensive epilepsy programme database. febrile seizures were defined as seizures occurring during a pyrexial illness between the age of 3 months and 5 years. ${ }^{24}$ In patients with seizures and a history of so called "meningitis" or "encephalitis", further hospital records were obtained and unless there was unequivocal evidence of an antecedent illness these cases were classed as febrile seizures. If there was a definite history of focal onset, more then one seizure in 24 hours, or a seizure lasting more than 15 minutes febrile seizures were classed as complex. ${ }^{24}$ Age of onset of first nonfebrile seizure and duration of epilepsy were recorded. Complex partial seizure frequency in the year preceding surgery was quantified as either daily, weekly, or monthly. Frequency of secondarily generalised seizures in the patient's lifetime was recorded as either never, once or less a year, or more than once a year.

CONTROLS

The controls were 98 age and randomly selected healthy volunteers (50 female, 48 male) with no history of head injury or significant medical or psychiatric illness. The mean age of the control group, 35.9 (SD 13.4) years (range 15-69), was not significantly different from the mean age of patients $(\mathrm{p}=0.4)$.

MAGNETIC RESONANCE IMAGING

Volumetric MRI was performed in all patients not more than 1 year before surgery. One hundred and twenty four coronal T1 weighted 1.5 $\mathrm{mm}$ contiguous slices were acquired on a 1.5 Tesla MRI scanner using a 3D spoiled gradient echo (SPGR) sequence. The flip angle was 20 degrees, matrix size $256 \times 192$, field of view $200 \times 150 \mathrm{~mm}$, with resultant voxel dimensions $0.78 \times 0.78 \times 1.50 \mathrm{~mm}$.

IMAGE ANALYSIS

Studies were transferred off line to an IBM RISC 6000 UNIX workstation using ANALYZE 7.55 (Mayo Foundation, MN 1995). All scans were reformatted to correct for tilt, so that the lateral fissures and longest length of fornix were as symmetric as possible. Hippocampal volume (HCV) was measured according to a previously published protocol..$^{25}$ Total brain volume (TBV) was measured using a 3D morphometric procedure. Significant hippocampal atrophy was defined as a volume less than 2 SD below the mean control volume, for each sex separately. Significant hippocampal asymmetry was defined as an $\mathrm{HCV}$ ratio (smaller/larger hippocampal volume) greater than 2 SD below the mean of controls. Correction of HCVs for TBVs was performed according to the covariance method described by Jack et $a l^{26}$ with the following equation:
HCV corrected $=\mathrm{HCV}$ measured gradient $\times(\mathrm{TBV}$ measured-TBV mean $)$

Gradient is the slope of the regression line of control HCV versus TBV, which was 0.0021 for male and 0.0015 for female subjects. This method has been validated by other workers. ${ }^{27}$

Table 1 Clinical variables in simple (SFS+), complex (CFS+), and no febrile seizures (FS-)

\begin{tabular}{llll}
\hline & $\begin{array}{l}S F S \\
(n=30)\end{array}$ & $C F S(n=7)$ & $F S-(n=40)$ \\
\hline Sex (F/M) & $20 / 10$ & $5 / 2$ & $21 / 19$ \\
Age at onset & $11.5(8.4)$ & $6.1(4.9)$ & $12.6(9.6)$ \\
Duration & $25.2(11.8)$ & $27.5(10.0)$ & $26.1(12.2)$ \\
Age at surgery & $36.0(9.3)$ & $34.4(9.6)$ & $38.7(8.7)$ \\
L/R TLE & $13 / 17$ & $5 / 2$ & $26 / 14$ \\
\hline
\end{tabular}

$\overline{\text { Values are mean (SD) unless otherwise stated. TLE=Temporal }}$ lobe epilepsy.

STATISTICAL ANALYSIS

The effect of a history of febrile seizures on hippocampal volumes was analysed by using one way analysis of variance (ANOVA) followed by a Tukey test for post hoc analysis, with the level of significance set at $p<0.05$. The relations between clinical variables and HCVs were examined with Pearson's correlation coefficients. The between rater coefficient of variability for HCV measurements was $10 \%$, consistent with previously published studies. ${ }^{28}$

\section{Results}

CONTROLS

In male subjects the mean (SD) $\mathrm{HCV}$ was 3421 (292) $\mathrm{mm}^{3}$ and the mean left HCV was 3349 (307) $\mathrm{mm}^{3}$. In female subjects the mean right $\mathrm{HCV}$ was 3088 (SD $326 \mathrm{~mm}^{3}$ and the mean left HCV was 3024 (SD 339) $\mathrm{mm}^{3}$. Hippocampal volumes were larger in male than in female subjects (two tailed Student's $t$ test $\mathrm{p}<0.01$ ). There was a small but significant right-left difference (two tailed Student's $t$ test $\mathrm{p}<0.01$ ), as found in previous studies ${ }^{27}$ Using a cut off of mean-2 SD, in female subjects the lower limit of normal for the right $\mathrm{HCV}$ was $2436 \mathrm{~mm}^{3}$ and the left HCV it was $2346 \mathrm{~mm}^{3}$. In male subjects the lower limit of normal for right $\mathrm{HCV}$ was $2837 \mathrm{~mm}^{3}$ and for the left HCV it was $2735 \mathrm{~mm}^{3}$. The mean $\mathrm{HCV}$ ratio (smaller/larger) in controls was 0.96 (SD 0.03).

PATIENTS

A history of febrile seizures was obtained in $48 \%$ of patients. There were 30 patients with a history of simple febrile seizures (SFS+), seven patients fulfilled the criteria for complex febrile seizures (CFS+), and 40 patients had no history of febrile seizures (FS-). Table 1 shows no difference in the age of onset of habitual seizures, duration of epilepsy, or age at the time of surgery, between any of these groups. In nine patients with febrile seizures, habitual seizures started after the febrile seizures and there was no identifiable latent period. Two of these patients had bilateral hippocampal atrophy. Of the 28 patients with a typical quiescent period in the evolution of their habitual seizures, the mean latent interval was 12.2 years (range 1-27). 


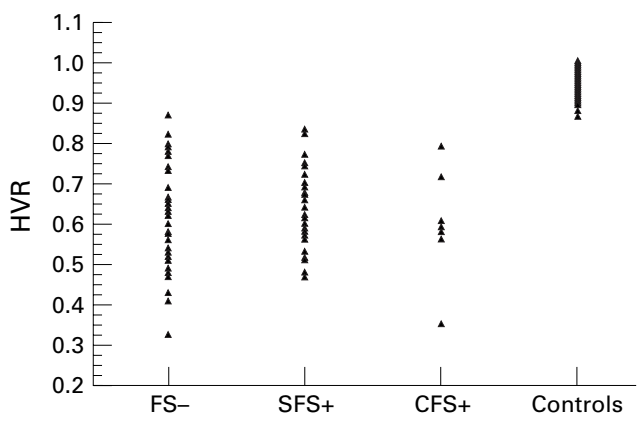

Figure 1 Hippocampal volume ratios in SFS+ patients (0.64 (SD 0.09), range 0.47-0.83), CFS+ patients $(0.60$ (SD 0.14), range 0.35-0.79) and FS- patients. (0.63 (SD 0.12), range 0.33-0.87) are significantly smaller than controls (0.95 (SD 0.03), range 0.87-1.00) $(p<0.001)$.

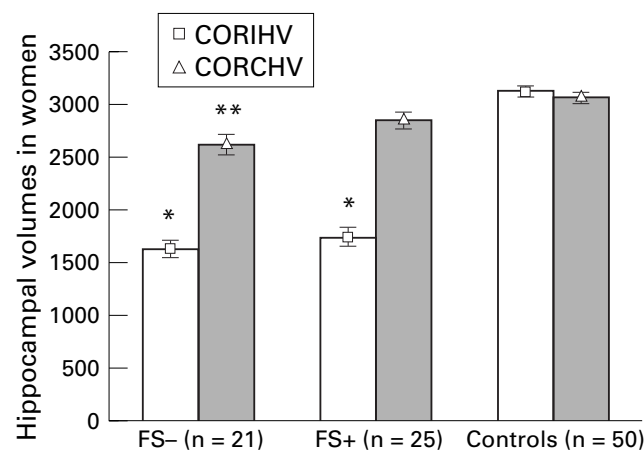

Figure 2 Female mean corrected hippocampal volumes $(S E)$, ipsilateral (CORIHV), and contralateral (CORCHV) to the seizure focus are not significantly different in FS+ and FS-patients. IHV is significantly smaller than controls $\left({ }^{*} p<0.01\right)$ in both groups. $C H V$ is significantly smaller than controls in the FS- group $\left(^{* *} p<\right.$ $0.01)$. Controls marked right $=C O R I H V$; left $=C O R C H V$. All volumes in $\mathrm{mm}^{3}$.

Hippocampal volume ratios avoid the inherent difference in absolute volumes between male and female subjects so that groups with unequal sex distribution can be compared. There was no difference in the degree of hippocampal asymmetry between SFS+, $\mathrm{CFS}+$ and FS- patients, but the three groups were all significantly smaller than controls (fig 1).

For absolute volumes males and female patients were considered separately. The CFS+ group was small (five female; two male) and therefore all SF+ patients were considered together. The mean corrected IHV and CHV were both larger in CFS+ patients (1921, 3089) than SFS+ patients (1688, 2684), despite a greater proportion of female patients.

In female patients $(n=46)$, ANOVA showed significant group differences in $\operatorname{IHV}(F(2$, 93 $)=1168, \mathrm{p}<0.01)$, and $\mathrm{CHV}(F(2,93)=8$, $\mathrm{p}<0.01)$. Post hoc analysis indicated no significant difference in IHV or CHV between FS+ and FS- groups. Both IHV in FS+ and FSgroups were smaller than controls $(p<0.01)$, but only the CHV in the FS- group was significantly smaller than controls $(\mathrm{p}<0.01)$ as shown in figure 2.

In male patients $(n=31)$, ANOVA showed significant group differences in ipsilateral (I) $(F$ $(2,76)=222, \mathrm{p}<0.01)$, and contralateral (C) $(F(2,76)=28, \mathrm{p}<0.01)$ HVs. Post hoc analysis

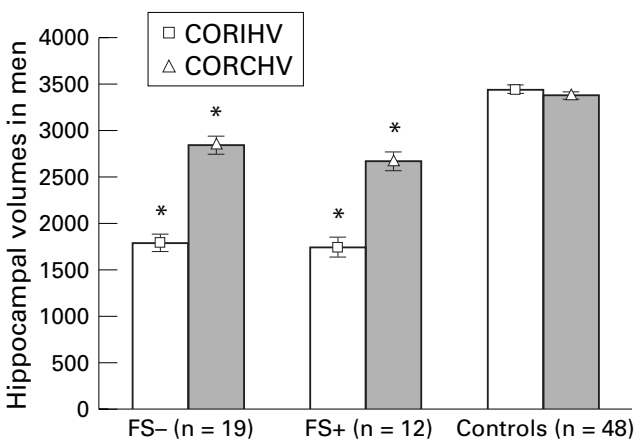

Figure 3 Male mean HCVs, (SE), IHVs, and CHVs to the seizure focus are not significantly different in FS+ and FS- patients. All volumes are significantly smaller than controls $\left({ }^{\star} p<0.01\right)$. Controls marked right $=C O R I H V$; left $=C O R C H V$. All volumes in $\mathrm{mm}^{3}$.

Table 2 Pearson's product moment correlations between hippocampal volumes and seizure variables

\begin{tabular}{|c|c|c|c|c|c|}
\hline Group & Side & $\begin{array}{l}\text { Age onset } \\
\text { seizures }\end{array}$ & $\begin{array}{l}\text { TLE } \\
\text { duration }\end{array}$ & $\begin{array}{l}\text { CPS } \\
\text { frequency }\end{array}$ & $\begin{array}{l}S G S \\
\text { frequency }\end{array}$ \\
\hline \multicolumn{6}{|c|}{ SFS+ $(n=30):$} \\
\hline & IHV & -0.03 & -0.10 & -0.31 & -0.02 \\
\hline & $\mathrm{CHV}$ & 0.11 & -0.25 & -0.16 & 0.26 \\
\hline \multicolumn{6}{|c|}{$\mathrm{CFS}+(\mathrm{n}=7)$ : } \\
\hline & IHV & -0.46 & $0.75^{\star}$ & 0.42 & 0.14 \\
\hline & $\mathrm{CHV}$ & -0.24 & 0.50 & 0.32 & 0.42 \\
\hline \multicolumn{5}{|c|}{ FS- $(n=40):$} & -0.02 \\
\hline & $\mathrm{CHV}$ & 0.17 & -0.14 & -0.21 & 0.02 \\
\hline
\end{tabular}

${ }^{\star} \mathrm{p}<0.05$. SFS $+=$ Simple febrile seizures; CFS $+=$ complex febrile seizures; FS $-=$ no febrile seizures; IHV=ipsilateral hippocampal volume; $\mathrm{CHV}=$ contralateral hippocampal volume; CPS=complex partial seizure; SGS=secondarily generalised seizure.

indicated no significant difference in either IHV or CHV between FS+ and FS- groups. All HCVs in male patients were significantly smaller than controls $(p<0.01)$ as shown in figure 3.

In all but one female patient the operated hippocampus was atrophic based on MR volumetry. Bilateral hippocampal atrophy was present in 27 patients (12 female, 15 male). Febrile seizures were no more common in patients with unilateral (23/50 patients, $46 \%$ ), than with bilateral hippocampal atrophy $(14 / 27$ patients, $52 \%$ ) (NS).

Table 2 shows Pearson's product moment correlations between HCVs and seizure variables for SFS+, CFS+, and FS- groups. Age of onset of seizures, duration of habitual epilepsy, or frequency of complex partial and secondarily generalised seizures did not correlate with HCVs in either the SFS+ or FS- groups. A paradoxical significant correlation existed between IHV and increasing duration of temporal lobe epilepsy in the CFS+ group.

\section{Discussion}

We found that MR determined hippocampal atrophy was no more severe in patients with temporal lobe epilepsy with a history of febrile seizures, either simple or complex, compared with those without such a history. This finding is surprising given previous reports. ${ }^{202123}$ Our study differs in that all our patients had pathologically proved hippocampal sclerosis, which is crucial if the hypothesis that febrile seizures might cause more severe hippocampal sclerosis 
is being tested. Given the recognised association of febrile seizures with hippocampal sclerosis it may be that, in studies without pathological confirmation, the FS+ patients are more likely than FS- patients to have typical hippocampal sclerosis as defined by $>50 \% \mathrm{CA} 1$ hippocampal cell loss. ${ }^{29}$

In the volumetric study by Cendes et al of 43 drug resistant patients with temporal lobe epilepsy, amygdala and hippocampal volumes ipsilateral to the seizure focus were significantly smaller in the 15 patients with a history of prolonged febrile seizures, compared with those without febrile seizures. ${ }^{2}$ Qualitative pathological study in 24 patients showed a higher proportion of more severe mesial temporal sclerosis in the group with prolonged febrile seizures, but this was not significant. Pathology was not reported in the remaining 19 patients.

Barr et al reported hippocampal volumetric findings in 44 patients undergoing evaluation for epilepsy surgery. ${ }^{21}$ Of the 21 patients (48\%) with febrile seizures, eight were "complex", five were " simple", according to standard criteria, and the remainder were unclassified. ${ }^{10}$ Hippocampal volume ipsilateral to the seizure focus was more than $2 \mathrm{SD}$ below the mean value in 12 of 18 FS+ patients, compared with four of 19 FS- patients. Pathological confirmation of hippocampal sclerosis was not provided, and only 16 of 44 patients had significant hippocampal atrophy on MRI. Given the minor degree of hippocampal atrophy in most FSpatients the possibility that they did not have typical hippocampal sclerosis cannot be excluded.

Theodore et al reported hippocampal volumes in 35 patients with uncontrolled complex partial seizures who had temporal lobe ictal onset on video-EEG. ${ }^{23}$ In nine patients with "prolonged or complex" febrile seizures ipsilateral hippocampal volumes were significantly smaller than those in the 26 patients without febrile seizures. Although 22 patients had surgery, pathology results were not provided, and again the possibility that they did not have typical hippocampal sclerosis must be considered.

The study of Kuks et al examined 107 patients with drug resistant epilepsy, hippocampal volume loss (defined as a hippocampal volume ratio $<0.96$ ) was more severe in those with childhood febrile seizures than those with non-febrile seizures and those without childhood seizures. ${ }^{20}$ All childhood febrile seizures were included. This study did not compare the degree of hippocampal volume loss in only patients with temporal lobe epilepsy with and without febrile seizures. Importantly, $64 \%$ of patients with hippocampal volume loss gave no history of febrile seizures, and as the authors emphasise, if febrile seizures do in some way contribute to hippocampal sclerosis, they cannot provide the only mechanism. Diffuse rather than focal hippocampal volume loss was more strongly associated with a history of febrile seizures, which led Kuks et al to propose that febrile seizures might convert pre-existing congenital focal abnormalities into diffuse hippocampal sclerosis.
Recent MRI findings in a study of familial febrile seizures add credence to this proposal. ${ }^{15}$ Fernandez et al did volumetric MRIs on 23 members of two families, of whom 13 had experienced febrile seizures and 10 had not. One member of each family with febrile seizures subsequently developed temporal lobe epilepsy. The 11 patients with febrile seizures who did not develop epilepsy and six clinically unaffected relatives had significantly asymmetric (>3 SD) hippocampi, the left always smaller. Previous surgical studies, as ours, have shown that febrile seizures are no more likely to be associated with left rather than right hippocampal sclerosis. ${ }^{36}$ These authors proposed that a subtle pre-existing hippocampal malformation might facilitate febrile seizures and contribute to the development of subsequent hippocampal sclerosis. Interestingly, in this study febrile seizures were predominantly simple, and in these two families with proposed unilateral hippocampal dysgenesis, no lateralised febrile seizures were described. Both cases of subsequent temporal lobe epilepsy had 25-50 simple febrile seizures, without any prolonged or focal febrile seizures. That localised hippocampal dysgenesis might predispose to simple febrile seizures questions the assumption that brains of infants with simple febrile seizures are normal, ${ }^{30}$ and refocuses interest on the relation between "simple" febrile seizures and later temporal lobe epilepsy.

Animal models provide a link between developmental abnormalities and susceptibility to seizures. In a rat hyperthermic model of febrile seizures, immature rats with experimentally induced neuronal migration defects had a lower threshold to hyperthermia induced seizures, ${ }^{17}$ and were more susceptible to irreversible hippocampal neuronal damage than control immature rats without migration defects. $^{18}$

\section{CLINICAL FINDINGS}

We found no significant correlations between clinical variables and hippocampal volumes in either the FS+ or FS- groups. These findings are consistent with earlier reports which showed no correlation between epilepsy duration or seizure frequency and hippocampal volumes with and without febrile seizures. ${ }^{19} 20$

Although Barr et al also found no significant correlations between duration of epilepsy, seizure frequency, and ipsilateral hippocampal volumes, a significant inverse correlation between seizure frequency and contralateral hippocampal volume in the FS+ group led the authors to propose that febrile seizures were associated with a smaller hippocampus ipsilateral to the seizure focus, whereas chronic factors were related to contralateral pathology. ${ }^{21}$ The second conclusion is based on a single -0.45 correlation coefficient between contralateral hippocampal volume in the FS+ group and seizure frequency. The clinical significance of this finding is undermined by the accompanying low $r$ value $(-0.14)$ for duration of chronic epilepsy in that group. We found no correlation between contralateral hippocampal volumes and seizure frequency. 
Theodore et al reported a correlation between duration of epilepsy and hippocampal volume ipsilateral to the seizure focus, in 35 patients with intractable temporal lobe epilepsy. ${ }^{23}$ This association remained significant even when the nine patients with prolonged febrile seizures were excluded. However, increasing duration of epilepsy was inversely associated with ipsilateral but not contralateral volumes, interpreted as suggesting that any deleterious effect of persistent seizures is confined to the epileptogenic zone itself.

The few patients in both studies might explain these opposite conclusions.

Only one of the 44 patients of Barr et al had significant bilateral hippocampal atrophy compared with 25 of 77 patients in our study. Of the 35 patients of Theodore et al there was a trend towards bilateral hippocampal atrophy in the FS+ but not FS- patients. Among our 77 patients febrile seizures were of a similar frequency in unilateral $(51 \%)$ and bilateral hippocampal atrophy $(40 \%)$. Our findings do not support the suggestion of Barr et al that febrile seizures contribute to ipsilateral atrophy, whereas chronic factors are related to contralateral pathology. ${ }^{21}$ Neither do our findings support the contrary proposition of Theodore et al that a trend towards bilateral hippocampal atrophy in FS+ patients is consistent with the effect of an early global insult.

We distinguished complex from simple febrile seizures. Previous studies have included only prolonged febrile seizures, ${ }^{23}$ but others have included all febrile seizures and still reported more severe hippocampal atrophy with febrile seizures. ${ }^{20}{ }^{21}$ The duration of febrile seizures is thought to be a major factor by those who argue that the seizures might cause hippocampal sclerosis ${ }^{141}$ but in these studies febrile seizures were unusually prolonged (>100 minutes). Such prolonged febrile seizures occur in $<1 \%$ of cases. ${ }^{32}$ The difficulty in distinguishing complex from simple febrile seizures in retrospective studies is well recognised, especially when a maternal history is not available. ${ }^{14}$ From the notes we were able to confirm complex febrile seizures in only eight patients, and these were analysed separately, but made no difference to the overall conclusions.

It is generally accepted that complex febrile seizures are associated with increased risk of temporal lobe epilepsy and simple febrile seizures with generalised epilepsies. ${ }^{33}$ HamatiHaddad et al examined a mixed population of all ages not all with longstanding epilepsy and found a strong association between febrile seizures and temporal lobe epilepsy. ${ }^{34}$ However, a prospective community based cohort study disputed a simple causal link between febrile seizures and hippocampal sclerosis. Of 524 children older than 1 year at the onset of epilepsy $73(13.9 \%)$ had febrile seizures and 33 of these were complex $(45.2 \%) ; 317$ had MRIs and definite hippocampal atrophy was found in only three, none of whom had febrile seizures. There was no evidence that prior febrile seizures were associated with complex partial seizures, temporal lobe epilepsy, or hippocam- pal atrophy. Our findings are consistent with these epidemiological data.

\section{Conclusions}

In this series of patients with surgically treated temporal lobe epilepsy with proved hippocampal sclerosis, $48 \%$ of patients had a history of febrile seizures. The severity of hippocampal atrophy determined by MRI is independent on a history of febrile seizures, both simple and complex, refuting the notion that such seizures cause more severe hippocampal sclerosis. Age of onset of first non-febrile seizure, temporal lobe epilepsy duration, and seizure frequency, both complex partial and secondarily generalised, did not correlate with the severity of hippocampal atrophy in patients with or without febrile seizures.

Our findings are consistent with the theory that hippocampal sclerosis may be a preexisting abnormality, possibly developmental, providing a substrate for febrile seizures in some patients. In retrospective analyses of patients with temporal lobe epilepsy, we caution ascribing pathoaetiological relevance to a history of febrile seizures, especially if not all patients are known to have definite hippocampal sclerosis.

1 Babb TL, Pretorius JK. Pathological substrates of epilepsy. In: Wylie E, ed. The treatment of epilepsy: principles and pracIn: Wylie E, ed. The treatment of epilepsy: principles

2 Cendes F, Andermann F, Dubeau F, et al. Early childhood prolonged febrile convulsions, atrophy and sclerosis of mesial structures, and temporal lobe epilepsy: an MRI volumetric study. Neurology 1993;43:1083-7.

3 French JA, Williamson PD, Thadani VM. Characteristics of medial temporal lobe epilepsy. 1. Results of history and physical examination. Ann Neurol 1993;34:774-80.

4 Abou-Khalil B, Andermann E, Andermann F. Temporal lobe epilepsy after prolonged febrile convulsions: excellent outcome after surgical treatment. Epilepsia 1993;34:878-

5 Berkovic SF, Andermann F, Olivier A, et al. Hippocampal sclerosis in temporal lobe epilepsy demonstrated by magnetic resonance imaging. Ann Neurol 1991;29:175-82.
Bruton C. The neuropathology of temporal lobe epilepsy. New York: Oxford University Press, 1988.

7 Falconer MA, Serafetinides EA, Corsellis JAN. Etiology and pathogenesis of temporal lobe epilepsy. Arch Neurol 1964;10:233-48.

8 Falconer MA, Taylor DC. Surgical treatment of drugresistant epilepsy due to mesial temporal sclerosis. Arch Neurol 1964;10:233-48.

9 Mathieson G. Pathology of temporal lobe foci. In: Penry JK, Daly DD, eds. Advances in neurology. New York: Raven Press, 1975:163-85.

10 Nelson KB, Ellenberg JH. Predictors of epilepsy in children who have suffered febrile seizures. N Engl F Med 1976;295: 1029-33.

11 Annegers JF, Hauser WA, Shirts SB. Factors prognostic of unprovoked seizures after febrile convulsions. $N$ Engl F Med 1987;316:493-8.

12 Verity CM, Golding J. Risk of epilepsy after febrile convulsions: a national cohort study. BMF 1991;303:1373-6.

13 LaBar KS, LeDoux JE, Spencer DD, et al. Impaired fear conditioning following unilateral temporal lobectomy in

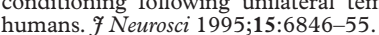

14 Maher JM, McLachlan RS. Febrile convulsions: Is seizure duration the most important predictor of temporal lobe duration the most important pre
epilepsy? Brain 1995;118:1521-8.

15 Fernandez G, Effenberger O, Vinz B, et al. Hippocampal malformation as a cause of familial febrile convulsions and subsequent hippocampal sclerosis. Neurology 1998;50:909-17.

6 Sperber EF. Age dependency of seizure-induced hippocampal dysfunction. In: Wolf $\mathrm{P}$, ed. Epileptic seizures and syndromes. London: John Libbey, 1994:469-80.

17 Germano IM, Zhang YF, Sperber EF, et al. Neuronal migration disorders increase susceptibility to hyperthermia-induced seizures in developing rats. Epilepsia 1996;37:902-10

18 Germano IM, Sperber EF, Ahuja S, et al. Evidence of enhanced kindling and hippocampal neuron injury in immature rats with neuronal migration disorders. Epilepsia 1998;39:1253-60.

19 Cendes F, Andermann F, Gloor P, et al. Atrophy of mesial structures in patients with temporal lobe epilepsy: cause or consequence of repeated seizures? Ann Neurol 1993;34: $795-801$. 
20 Kuks JBM, Cook MJ, Fish DR, et al. Hippocampal sclerosis in epilepsy and childhood febrile seizures. Lancet 1993;342: in epilepsy

21 Barr WB, Ashtari M, Schaul N. Bilateral reductions in hippocampal volume in adults with epilepsy and a history of febrile seizures. F Neurol Neurosurg Psychiatry 1997;63:4617.

22 Trenerry MR, Jack $\mathrm{CR}$, Sharbrough FW, al Quantitative MRI hippocampal volumes: association with onset and duration of epilepsy, and febrile convulsions in tempora lobectomy patients. Epilepsy Res 1993;15:247-52.

23 Theodore WH, Bhatia S, Hatta J, et al. Hippocampal atrophy, epilepsy duration, and febrile seizures in patients with partial seizures. Neurology 1999;52:132-6.

24 NIH Consensus Development Conference Summary. Consensus statement on febrile seizures. In: Nelson KB, Ellenberg JH, eds. Febrile seizures. New York: Raven Press, 1981: berg J

25 Cook MJ, Fish DR, Shorvon SD, et al. Hippocampal volumetric and morphometric studies in frontal and volumetric and morphometric studies in frontal lobe epilepsy. Brain 1992;115:1001-15.

26 Jack CR, Colleen KT, Zinsmeister AR, et al. Anterior temporal lobes and hippocampal formations: normative volumetric measurements from MR images in young adults. Radiology 1989;172:549-54.
27 Free SL, Bergin PS, Cook MJ, et al. Methods for normalisation of hippocampal volumes measured with MR. Am $\mathcal{F}$ Neuroradiol 1995;16:637-43.

28 Van Paesschen W. The spectrum of hippocampal sclerosis:a quantitative magnetic imaging study. Ann Neurol 1997;41: $41-51$.

29 Sagar HJ, Oxbury JM. Hippocampal neuron loss in temporal lobe epilepsy: correlation with early childhood convulsions. Ann Neurol 1987;22:334-40.

30 Sloviter RS, Pedley TA. Subtle hippocampal malformations: importance in febrile seizures and development of epilepsy [editorial]. Neurology 1998;50:846-9.

31 Van Landingham KE, Heinz ER, Cavazos JE, et al. Magnetic resonance imaging evidence of hippocampal injury after prolonged febrile convulsions. Ann Neurol 1998;43:413-26.

32 DeLong GR, Hienz ER. The clinical syndrome of early-life bilateral hippocampal sclerosis. Ann Neurol 1999;42:11-17.

33 Berkovic SF, Scheffer IE. Febrile seizures: genetics and relationship to other epilepsy syndromes. Curr Opin Neurol 1998;11:129-34.

34 Hamati-Haddad A, Abou-Khalil B. Epilepsy diagnosis and localization in patients with antecedent childhood febrile convulsions. Neurology 1998;50:917-22. 